\title{
An apparent slip mechanism between two spheres based on solvent rheology: theory and implication on the shear-thinning of non-Brownian suspensions
}

\author{
A. Vázquez-Quesada* \\ Zienkiewicz Centre for Computational Engineering (ZCCE), \\ Swansea University, Bay Campus, Swansea SA1 8EN, United Kingdom. and \\ Department of Theoretical Condensed Matter Physics, \\ Universidad Autónoma de Madrid, 28049 Madrid, Spain. \\ Pep Español ${ }^{\dagger}$ \\ Departamento de Física Fundamental, UNED, \\ Apartado 60141, Madrid 28080, Spain \\ M. Ellero $\ddagger$ \\ Basque Center for Applied Mathematics (BCAM), \\ Alameda de Mazarredo 14, 48400 Bilbao, Spain. \\ IKERBASQUE, Basque Foundation for Science, \\ Calle de María Díaz de Haro 3, 48013 Bilbao, Spain and \\ Zienkiewicz Centre for Computational Engineering (ZCCE), \\ Swansea University, Bay Campus, Swansea SA1 8EN, United Kingdom.
}

(Dated: November 13, 2018) 


\begin{abstract}
Analytical results for the apparent slip between two spheres in a simple biviscous model of a shear thinning fluid are presented. Velocity profiles and apparent slip lengths along the surfaces are analyzed in order to characterize the physical mechanism. It is shown that in this non-Newtonian model, the effect of shear-thinning limited to high-shear rates in the interstitial regions between close spheres can be alternatively interpreted as the onset of an apparent shear-rate dependent slippage effect. The results of the theory compare well with experiments from the literature showing the presence of surface slip on a particle approaching a planar wall. In terms of implications on suspensions rheology, the present results bridge the 'hidden' solvent shear-thinning theory [A. Vázquez-Quesada et al. , Phys. Rev. Lett., 117, 108001-5 (2016)] with slip-based models presented recently in [M. Kroupa et al., Phys. Chem. Chem. Phys. 19, 5979-5984 (2017)] as a possible explanation on the mechanism behind the shear-thinning in hard-sphere non-Brownian suspensions.
\end{abstract}

\footnotetext{
* adolfo.vazquez@uam.es

$\dagger$ pep@fisfun.uned.es

$\ddagger$ mellero@bcamath.org
} 


\section{INTRODUCTION}

The shear-thinning behaviour observed in concentrated non-Brownian suspension of spheres represents a long-standing issue in rheology [1-4]. In fact, in absence of Brownian and inertial effects -and if a simple Newtonian solvent is considered- the resulting bulk suspension viscosity should be constant [5] which is in contradiction with experimental results.

Three main hypothesis have been advanced in order to explain the observed Shear Thinning of Non-Brownian Suspensions (STNBS): an effective size of the particles that is reduced with increasing shear, shear thinning of the solvent itself at the high shear rates occurring at interstitial regions, and slip at the surface spheres.

Advocating the first hypothesis, some authors have recently associated the STNBS to the fact that the system behaves essentially as a suspension of soft particles at low shear stresses, with an apparent size that includes both the hard sphere and a part of the surrounding soft repulsive potential [6]. In simulations performed by Mari et al. [7] for example, the value of the repulsive length in the interparticle potential is chosen in the order of $5 \%$ of the particle radius $a$ (i.e. the so-called Debye length $\kappa^{-1}=0.05 a$ ) corresponding to typical non-zero force contributions on a spatial range of order of $0.1 \mu \mathrm{m}$ for particles a few $\mu \mathrm{m}$ in size. As discussed recently by Chatte et al. [6], under these conditions the 'apparent' size of the particles (including hard-core and soft repulsive range) will decrease with increasing shear rate leading to a suspension viscosity which decreases as a function of the shear rate, i.e. shear-thinning.

Previous results [8] had also shown that excluded volume effects can induce a shearthinning contribution coming from interparticle repulsive forces, however this was significant only for soft particles. In the hard-sphere limit, the interparticle contribution to the suspension viscosity was found irrelevant. This was also confirmed by independent nonBrownian simulations using nearly hard-core potentials (i.e. small effective repulsive ranges $\left.\kappa^{-1}=0.001 a\right)$ where Newtonian or opposite mild shear-thickening behaviour was always reported [9-11]. It is worth noticing that in most of the non-colloidal systems studied in the literature where shear-thinning has been observed [1-4], the particle size exceeds typically $40 \mu \mathrm{m}$, and shear-thinning was observed for surface roughness down to 30-90 nm [12], i.e. in the hard-sphere regime discussed above. Therefore, for hard-sphere non-colloidal systems 
shear-thinning is unlikely to be due to an excluded volume effect.

The second hypothesis, advanced in a recent work [13], suggests that in the interstitial regions between spherical particles very high shear rates can occur, which can trigger a local shear-thinning of a 'nominally' Newtonian matrix; this effect, in turn, can lead to shear thinning at the average (macroscopic) shear rate. Since the matrix shear-thinning is negligible at the mean shear rate and the local thinning occurs well beyond, the matrix was considered as 'nominally' Newtonian and the mechanism was termed as 'hidden'. Good agreement between experiments and simulations for suspensions of non-colloidal particles in several silicon oils was obtained. It should be noticed that in the case of simpler liquids (e.g. glycerol etc.), although shear-thinning does take place at extremely large critical bulk shear rates, hydrodynamic conditions under strong confinement can alter the liquid property itself. In the work by Jabbarzadeh et al. [14], for example, molecular simulations have shown that extreme confinement (in the order of tens molecular sizes) -similar to that encountered in the squeezing flow between nearly touching surfaces- can anticipate significantly the onset of matrix shear-thinning with respect to the bulk predictions, and therefore this effect might play a role even for liquids which are more Newtonian than lowmolecular-weight oils. This was also shown in experiments with other simple lubricating fluids, such as octamethylcyclotetrasiloxane (OMCTS), where a massive increase in the viscosity was observed as a result of liquid-solid [15-17] or continuous glass-like transitions $[18,19]$. In all these cases, the effective liquid shear-thinning is the result of an increased viscosity with respect to the bulk value linked to an alteration of its microstructure in response to strong confinement and generally disappear for liquid film thickness beyond 10 molecular dimensions [20, 21].

Finally, very recently a new mechanism has been proposed for non-colloidal shear-thinning based on the connection between the slip on the surface of the particles and the suspension viscosity [22]. Results based on a phenomenological slip model showed that surface slip might be crucial for the rheology and flow in crowded systems and can be an alternative explanation for STNBS. Traditionally, when a deviation of the no-slip behavior is detected, a constant slip length is generally used to characterize the slip on the experiment. Such a length is calculated a posteriori from the results of the experiment, so it is not used to do predictions but rather to calibrate the system behaviour [23]. In Ref. [22] a phenomenological relation between slip length and local shear rate in the gap between two particle was assumed. 
In fact, slip between fluids and solids has been observed in many experiments [24] and reported, for example, in concentrated polymer suspensions and polymer melts [25, 26]. Different explanations and theories have been proposed related to the microstructural changes of polymer configuration of the fluid close to the solid interface [27]. Shear dependent wall slip has been observed even in Newtonian (low-molecular-weight) liquids [20, 21, 28], and it can be associated to several factors including surface properties (wetting, rugosity, dissolved gas layer, cavitation) [29-33], as well as bulk liquid effects including apparent shear-thinning at large shear-rates or viscous heating $[34,35]$. It should be remarked that the effective shear-thinning of the liquid related to a slippage effect is intrinsically different from the confinement-induced effect discussed above. In fact in that case the effect disappear for wider lubricating films, whereas effective shear-thinning (e.g. reduced lubrication force) is still observed in experiments [20, 21].

In this work, we take the point of view that an apparent slip at the surface of the particles may be due to shear-banding effects, i.e. a decrease in viscosity in a very thin near-to-wall layer due to either a change in the structure of liquid, enhanced nucleation of a dissolved gas $[23,28,31]$, or a shear thinning of the solvent itself [36]. We reformulate some of the conclusions made in the 'hidden' shear-thinning approach of Ref. [13] in terms of an apparent slip length occurring in the interstitial squeezing flow between particles and show that this interpretation is consistent with the arguments made by Kroupa et al. [22].

Specifically, we present a study of the velocity profiles of the squeezing flow of a shear thinning fluid between two spheres in the lubrication regime, when the gap between the spheres is much smaller than their radii. To allow for an analytical treatment, the solvent is modelled as a simple biviscous fluid. The model developed in [37] allows us to calculate the resulting 'apparent' slip-length which is well-defined, exhibits a natural dependence on the local shear-rate and has a one-to-one correspondence to the underlying liquid rheology.

The results show that 'hidden' shear-thinning models for nominally-Newtonian matrices such as those presented in Ref. [13] can be alternatively interpreted in terms of an 'apparent' surface slip. This leads to an equally possible explanation of the shear-thinning mechanism on non-colloidal systems in agreement with the one put forward recently by Kroupa et al.[22]. 


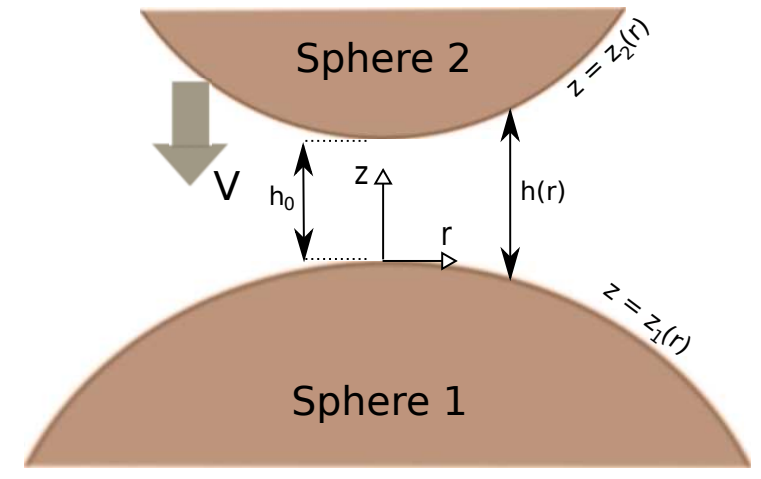

FIG. 1. Scheme of two approaching spheres of radii $a_{1}, a_{2}$ in the lubrication regime $\left(h_{0} \ll a_{1}, a_{2}\right)$.

\section{BIVISCOUS MODEL: SQUEEZING FLOW}

In this section, we present a summary of the relevant analytical results for the velocity profile, pressure gradient, and force in the squeezing flow between two very close spheres embedded in a biviscous fluid. Further details can be found in Ref. [37]. The fluid is assumed to obey the following constitutive equation for the shear viscosity

$$
\eta=\left\{\begin{array}{ll}
\eta_{0}, & \text { if }|\dot{\gamma}|<\dot{\gamma}_{c} \\
\eta_{1}, & \text { if }|\dot{\gamma}| \geq \dot{\gamma}_{c}
\end{array} \quad \text { with } \quad \eta_{0}>\eta_{1}\right.
$$

That is, for shear rates below the critical shear rate $\dot{\gamma}_{c}$ the fluid has a large viscosity $\eta_{0}$, while it has a small viscosity $\eta_{1}$ for larger shear rates. This is a very schematic rheological model that nevertheless captures the basic phenomenology of a shear thinning fluid. Because of the simplicity of the model, analytical solutions can be found.

We consider the flow problem in which two spheres of radii $a_{1}$ and $a_{2}$ separated by a minimum gap $h_{0}$ approach each other with a normal relative velocity $V$. The flow problem in which the two spheres move with parallel and opposite velocities thus generating a shear flow is not considered here because it gives much smaller velocity profiles. The system of reference and the different parameters of the problem are shown in Fig. 1. When the velocity $V$ is small enough and the gap is small, we may assume the validity of the lubrication regime, where the fluid squeezes uni-directionally along the $r$ coordinate. Under these conditions, the Stokes equations with no-slip boundary conditions plus continuity of stress along the gap can be solved. In the case of a biviscous fluid, the model can display a position $r$ dependent shear banding, that is, depending on critical conditions the fluid displays two regions denoted as $\mathcal{R}_{m}, \mathcal{R}_{b}$ along the horizontal direction. The flow field has no discontinuities in the shear 
rate in $\mathcal{R}_{m}$ (mono-viscous-like region) whereas in region $\mathcal{R}_{b}$ (biviscous-like region) the fluid does shear-band. As discussed in Ref. [37], these regions $\mathcal{R}_{m}$ and $\mathcal{R}_{b}$ are defined as

$$
\begin{aligned}
& r \in \mathcal{R}_{m} \text { if } r \leq r_{1} \text { or } r \geq r_{2} \\
& r \in \mathcal{R}_{b} \text { if } r_{1}<r<r_{2}
\end{aligned}
$$

where $r_{1}$ and $r_{2}$ are given by

$$
\begin{aligned}
& r_{1}=\mathcal{Q}-\sqrt{-3-\mathcal{Q}^{2}+4\left(\frac{\dot{\gamma}_{s}^{\max }}{\dot{\gamma}_{c}}\right) \frac{1}{\mathcal{Q}}} \\
& r_{2}=\mathcal{Q}+\sqrt{-3-\mathcal{Q}^{2}+4\left(\frac{\dot{\gamma}_{s}^{\max }}{\dot{\gamma}_{c}}\right) \frac{1}{\mathcal{Q}}}
\end{aligned}
$$

with

$$
\begin{aligned}
& \mathcal{Q}=\sqrt{-1+\frac{1}{\mathcal{P}}+\mathcal{P}} \\
& \mathcal{P}=\sqrt[3]{-1+2\left(\frac{\dot{\gamma}_{s}^{\text {max }}}{\dot{\gamma}_{c}}\right)^{2}+2\left(\frac{\dot{\gamma}_{s}^{\text {max }}}{\dot{\gamma}_{c}}\right) \sqrt{\left(\frac{\dot{\gamma}_{s}^{\text {max }}}{\dot{\gamma}_{c}}\right)^{2}-1}}
\end{aligned}
$$

and

$$
\dot{\gamma}_{s}^{\max }=\frac{9}{8} \frac{V}{h_{0}} \sqrt{\frac{3}{2} \frac{a}{h_{0}}}
$$

where $a=\left(1 / a_{1}+1 / a_{2}\right)^{-1}$ is the reduced radius. The fluid shear bands, i.e. $\mathcal{R}_{b} \neq \emptyset$, when the following condition for the interparticle separation distance is satisfied

$$
h_{0}<h_{0}^{\lim }
$$

where

$$
h_{0}^{\lim } \equiv \frac{3}{4} \sqrt[3]{\frac{9 V^{2} a}{2 \dot{\gamma}_{c}^{2}}}
$$

This condition is equivalent to

$$
\dot{\gamma}_{c}<\dot{\gamma}_{s}^{\max }
$$

In other words, given a bead radius $a$ and a fluid rheology $\dot{\gamma}_{c}$, there is a critical distance $h_{0}^{\text {lim }}$, determined by the approaching velocity $V$ such that, for smaller separations $h_{0}$, the flow shows two distinct regions along the $r$ direction, a shear banded one $\mathcal{R}_{b}$ with discontinuities 
separating two fluids with different viscosities, and another one $\mathcal{R}_{m}$ without velocity-gradient discontinuities.

The corresponding flow field is given by the following piece-wise two-dimensional velocity profile

$$
u(r, z)=\left\{\begin{array}{c}
\left.u(r, z)\right|_{m}, \text { if } r \in \mathcal{R}_{m} \\
\left.u(r, z)\right|_{b}, \text { if } r \in \mathcal{R}_{b}
\end{array}\right.
$$

The functional form of $\left.u(r, z)\right|_{m}$ and $\left.u(r, z)\right|_{b}$ is

$$
\begin{aligned}
\left.u(r, z)\right|_{m} & =\frac{1}{2 \eta_{0}} \frac{\partial p}{\partial r}(r)\left(z-z_{1}(r)\right)\left(z-z_{2}(r)\right) \\
\left.u(r, z)\right|_{b} & =\left\{\begin{array}{cc}
\frac{1}{2 \eta_{0}} \frac{\partial p}{\partial r}(r)\left[(z-c(r))^{2}-z_{c}^{2}(r)\right]+\frac{1}{2 \eta_{1}} \frac{\partial p}{\partial r}(r)\left[z_{c}^{2}(r)-h^{2}(r) / 4\right], & \text { if }|z-c(r)|<z_{c}(r) \\
\frac{1}{2 \eta_{1}} \frac{\partial p}{\partial r}(r)\left(z-z_{1}(r)\right)\left(z-z_{2}(r)\right), & \text { if }|z-c(r)| \geq z_{c}(r)
\end{array}\right.
\end{aligned}
$$

Here, $c(r)=\left(z_{1}(r)+z_{2}(r)\right) / 2$ is the loci of points equidistant from both surfaces. At a given value of $r \in \mathcal{R}_{b}$, the velocity profile has two different regions: in $|z-c(r)| \leq z_{c}(r)$ the effective viscosity is $\eta_{0}$, while in $|z-c(r)|>z_{c}(r)$ it is $\eta_{1}$. The distance $z_{c}(r)$ is given, when $r \in \mathcal{R}_{b}$, as

$$
\begin{aligned}
& \left(\frac{2 z_{c}(r)}{h(r)}\right)=S_{1}+S_{2} \\
& S_{1}=\sqrt[3]{\frac{\eta_{0}}{2\left(\eta_{0}-\eta_{1}\right)}\left(1+\sqrt{1+\frac{4}{27} \frac{\eta_{1}^{2}}{\eta_{0}^{2}} \frac{\eta_{1}}{\eta_{0}-\eta_{1}}\left(\frac{\dot{\gamma}_{s}(r)}{\dot{\gamma}_{c}}\right)^{3}}\right)} \\
& S_{2}=\sqrt[3]{\frac{\eta_{0}}{2\left(\eta_{0}-\eta_{1}\right)}\left(1-\sqrt{1+\frac{4}{27} \frac{\eta_{1}^{2}}{\eta_{0}^{2}} \frac{\eta_{1}}{\eta_{0}-\eta_{1}}\left(\frac{\dot{\gamma}_{s}(r)}{\dot{\gamma}_{c}}\right)^{3}}\right)}
\end{aligned}
$$

where $h(r)$ is the gap size at the coordinate $r$ and $\dot{\gamma}_{s}(r)=3 r V / h^{2}(r)$ is the maximum shear rate that a Newtonian fluid would have at the given position $r$. This maximum would occur at the surface of the spheres.

The value $\dot{\gamma}_{s}^{\max }$ in Eq. (5) is the maximum of the function $\dot{\gamma}_{s}(r)$. Note that $z_{c}(r)$ is a distance which can not be greater than $h(r) / 2$ when $r \in \mathcal{R}_{b}$. When the gap between the two spheres is much smaller than the radii of the spheres, the distance between the two surfaces is given approximately by $h(r)=h_{0}\left(1+\frac{r^{2}}{2 a h_{0}}\right)$. 
The pressure gradient for $r \in \mathcal{R}_{b}$ is

$$
\left.\frac{\partial p}{\partial r}(r)\right|_{b}=-\frac{\dot{\gamma}_{c} \eta_{0}}{z_{c}(r)}
$$

In the region $\mathcal{R}_{m}$ the pressure gradient is given by the usual expression for a Newtonian fluid

$$
\left.\frac{\partial p}{\partial r}(r)\right|_{m}=-\frac{6 \eta_{0} r V}{h^{3}(r)}
$$

Finally, an approximated analytical expression of the force between both spheres is given by $[37]$

$$
F= \begin{cases}F_{1} & \text { if } h_{0} \geq h_{0}^{l i m} \\ F_{2} & \text { if } h_{0}<h_{0}^{l i m}\end{cases}
$$

where

$$
\begin{aligned}
F_{1}= & 6 \pi \eta_{0} V a^{2} h_{0}^{-1} \\
F_{2}= & 2 \pi\left[3 a^{2} \eta_{0} V h_{0}^{-1}+3 a^{2}\left(\eta_{0}-\eta_{1}\right) V\left(h^{-1}\left(r_{2}\right)-h^{-1}\left(r_{1}\right)\right)\left(2-h_{0}\left(h^{-1}\left(r_{1}\right)+h^{-1}\left(r_{2}\right)\right)\right)+\right. \\
& \left.2 a \dot{\gamma}_{c}\left(\eta_{0}-\eta_{1}\right)\left[\left(r_{2}-r_{1}\right)+\left(h\left(r_{1}\right)-2 h_{0}\right) \sqrt{\frac{2 a}{h_{0}}}\left(\arctan \left(\frac{r_{2}}{\sqrt{2 a h_{0}}}\right)-\arctan \left(\frac{r_{1}}{\sqrt{2 a h_{0}}}\right)\right)\right]\right]
\end{aligned}
$$

\section{SLIP MECHANISM}

\section{A. Conditions for slip between spheres}

Although from a theoretical perspective slip and shear banding are different phenomena, from an experimental point of view, a shear banding fluid may produce a behaviour that is indistinguishable from slip of a usual Newtonian fluid. In this section, we re-interpret the results of a shear banding biviscous fluid in terms of an apparent slip of a Newtonian fluid with a viscosity given by $\eta_{0}$.

Note that the expressions for $r_{1}$ and $r_{2}$ in Eq. (3) do not depend on the viscosity ratio $\eta_{1} / \eta_{0}$. This implies that the region $\mathcal{R}_{b} \neq \emptyset$ as long as the condition (8) holds. In this case, a boundary layer of viscosity $\eta_{1}$ on the surfaces of the spheres between the coordinates $r_{1}$ and $r_{2}$ is always present. From Eq. (11) the following limit can be calculated

$$
\lim _{\eta_{1} / \eta_{0} \rightarrow 0} z_{c}(r)=\frac{h(r)}{2}
$$


indicating that such a layer becomes infinitesimal in such a limit. Therefore, in the limit $\eta_{1} / \eta_{0} \rightarrow 0$, if (8) is satisfied, there will be a real slip in the region $\mathcal{R}_{b}$.

The other parameter controlling $z_{c}(r)$ is $\dot{\gamma}_{c}$; in the limit $\dot{\gamma}_{c} \rightarrow 0$ one obtains that $r_{1} \rightarrow 0$, $r_{2} \rightarrow \infty$ and $h_{0}^{\lim } \rightarrow \infty, z_{c}(r) \rightarrow 0$ and the behavior of a solvent of viscosity $\eta_{1}$ is recovered. Therefore, for small $\dot{\gamma}_{c}$, the layer of the fluid of viscosity $\eta_{1}$ close to the spheres can not be considered as a thin layer, given that the thickness of such a layer is comparable to the gap. In conclusion, an apparent slip can be observed if i) the condition (8) is satisfied, ii) the critical shear rate $\dot{\gamma}_{c}$ is high enough, i.e, $\dot{\gamma}_{c} / \dot{\gamma}_{s}\left(r_{\max }\right)$ is not too small, and iii) the viscosity ratio $\eta_{1} / \eta_{0}$ is low enough. The last two conditions can be reduced to $z_{c}(r) \sim h(r) / 2$ at some $r \in\left[r_{1}, r_{2}\right]$, which is the condition of having a thin layer.

Of course, the proposed conditions are only necessary to detect slip if the velocity profiles are observed directly. If the slip is inferred from indirect methods, any deviation from the Newtonian behavior could be interpreted as slip, making the first condition as the only necessary one.

\section{B. Calculation of the slip length}

Under the above conditions, a slip behavior will be present between both spheres in the region $\mathcal{R}_{b}$, this is in $r_{1}<r<r_{2}$. The apparent slip length $\lambda$ is defined as the distance beyond the interface at which the liquid velocity extrapolates to zero and it can be calculated in that region from the analytical velocity profile (10).

If we consider that the region $|z-c(r)| \geq z_{c}(r)$ is very narrow, the apparent velocity $u_{a p}$ in the surface of the spheres will be the extension of the velocity profile in the region $|z-c(r)|<z_{c}(r)$ to the coordinates $z=z_{1}(r)$ or $z=z_{2}(r)$. The expression is

$$
u_{a p}=\left.\frac{1}{2} \frac{\partial p}{\partial r}(r)\right|_{b}\left(\frac{1}{\eta_{1}}-\frac{1}{\eta_{0}}\right)\left[z_{c}^{2}(r)-h^{2}(r) / 4\right]
$$

Within the lubrication regime, given that $a \gg r$, curvature effects do not enter into the slip length, which can be calculated as

$$
\lambda(r)=\frac{u_{a p}(r)}{\dot{\gamma}^{a p}(r)}
$$

where the apparent shear rate $\dot{\gamma}^{a p}(r)$ on the coordinates $z=z_{1}(r)$ or $z=z_{2}(r)$ is given by

$$
\dot{\gamma}^{a p}(r)=-\left.\frac{1}{\eta_{0}} \frac{\partial p}{\partial r}(r)\right|_{b} \frac{h(r)}{2}=\dot{\gamma}_{c}\left(\frac{h(r)}{2 z_{c}(r)}\right)
$$


The final result is

$$
\lambda(r)=\frac{h(r)}{4}\left(\frac{\eta_{0}}{\eta_{1}}-1\right)\left[1-\left(\frac{\dot{\gamma}_{c}}{\dot{\gamma}_{a p}(r)}\right)^{2}\right]
$$

where the relation of the slip length with $\dot{\gamma}_{a p}(r)$ has been explicitly written. Note that the slip length in the present model depends on the coordinate $r$, at variance with usual approximations in which it is taken as a constant [28, 38, 39].

Note also that when the limit $\eta_{1}=\eta_{0}$ is considered, although the length $\lambda$ can not be considered properly as a slip length because the condition $\eta_{1} / \eta_{0}<<1$ is not held, the no-slip condition is recovered because $\lim _{\eta_{1} \rightarrow \eta_{0}} \lambda(r)=0$. In the opposite limit, when $\eta_{1} / \eta_{0} \rightarrow 0$, the analytical expression for the slip length tends to

$$
\lim _{\eta_{1} / \eta_{0} \rightarrow 0} \lambda(r)=\frac{h(r)}{6}\left(\frac{\dot{\gamma}_{s}(r)}{\dot{\gamma}_{c}}-1\right)
$$

i.e. it is proportional to the wall shear rate leading to higher apparent fluid slippage on regions of higher local wall shear rates. Note that Eqs. (20) and (21) are valid only in the

biviscous regions. In the monoviscous regions, i.e. for $\dot{\gamma}_{s}(r) \leq \dot{\gamma}_{c}$, there is not apparent slip, and the slip length is 0 .

\section{Slip length: model parameters sensitivity}

In order to illustrate the above theoretical results in more practical terms, we consider the typical case of two non-colloidal spherical particles of radius $R=50 \mu \mathrm{m}$ approaching each other along the line joining their centers at a relative velocity $V=a \dot{\gamma}$. We investigate the effect of changing the ratio $\eta_{1} / \eta_{0}$ within the range $0.01-0.9$ while keeping the following parameters constant: $\dot{\gamma}=1 \mathrm{~s}^{-1}, \dot{\gamma}_{c u t}=1000 \mathrm{~s}^{-1}$ and $h_{0}=4 \times 10^{-3} R$.

In Fig. 2 (top) we plot the velocity profiles at the coordinate $r=\left(r_{1}+r_{2}\right) / 2$. As already mentioned, $r_{1}$ and $r_{2}$ do not depend on the ratio $\eta_{1} / \eta_{0}$ so they remain the same for all velocity profiles. When the ratio $\eta_{1} / \eta_{0}$ is decreased the profile tends to a slip behavior in the region $\mathcal{R}_{b}$, determined by the limit (21) of the slip length. Even at the largest ratios one can calculate an 'apparent' slip length for all the profiles via Eq. (20). Such slip lengths are shown, for the whole region $\mathcal{R}_{b}$, in Fig. 2 (bottom). Note that, although $r_{1}$ can be very 

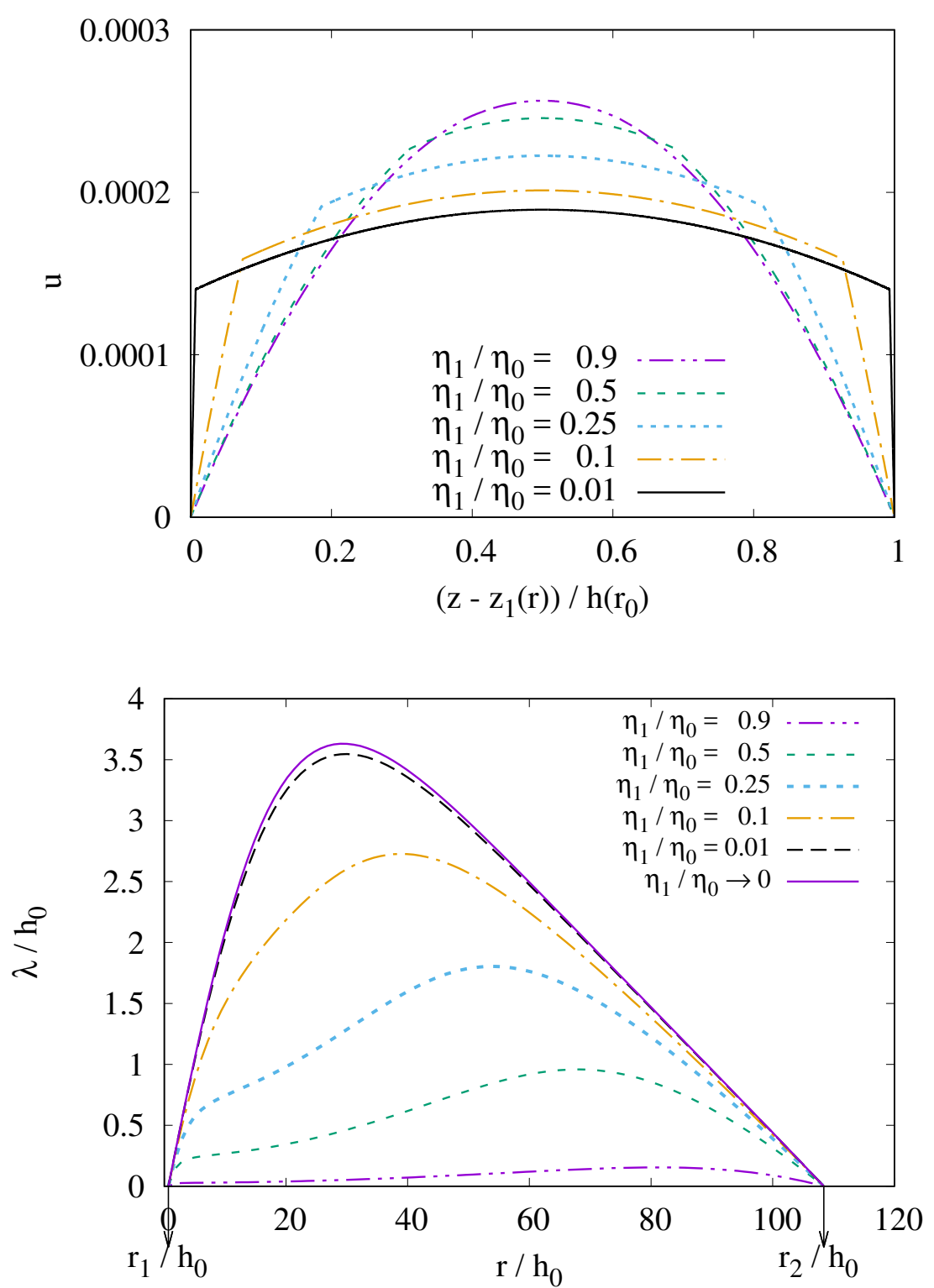

FIG. 2. Top: velocity profiles for different ratios $\eta_{1} / \eta_{0}$ at $r=\left(r_{1}+r_{2}\right) / 2$. Bottom: slip lengths of the same systems at region $r_{1}<r<r_{2}$ calculated via Eq. (20). The limiting curve (21) for $\eta_{1} / \eta_{0} \rightarrow 0$ case has also been drawn.

small, as in the figure, it is not zero, so in this model, there will be always a region around $r=0$ free of apparent slip.

The same system is considered now at a fixed viscosity ratio $\eta_{1} / \eta_{0}=10^{-2}$ with a varying dimensionless gap between the spheres $h_{0}^{*}=h_{0} / h_{0}^{\text {lim }}$ within the range $0.01-0.9$. Given that now the values of $r_{1}$ and $r_{2}$ are changing with $h_{0}$, the velocity profiles are drawn at the 

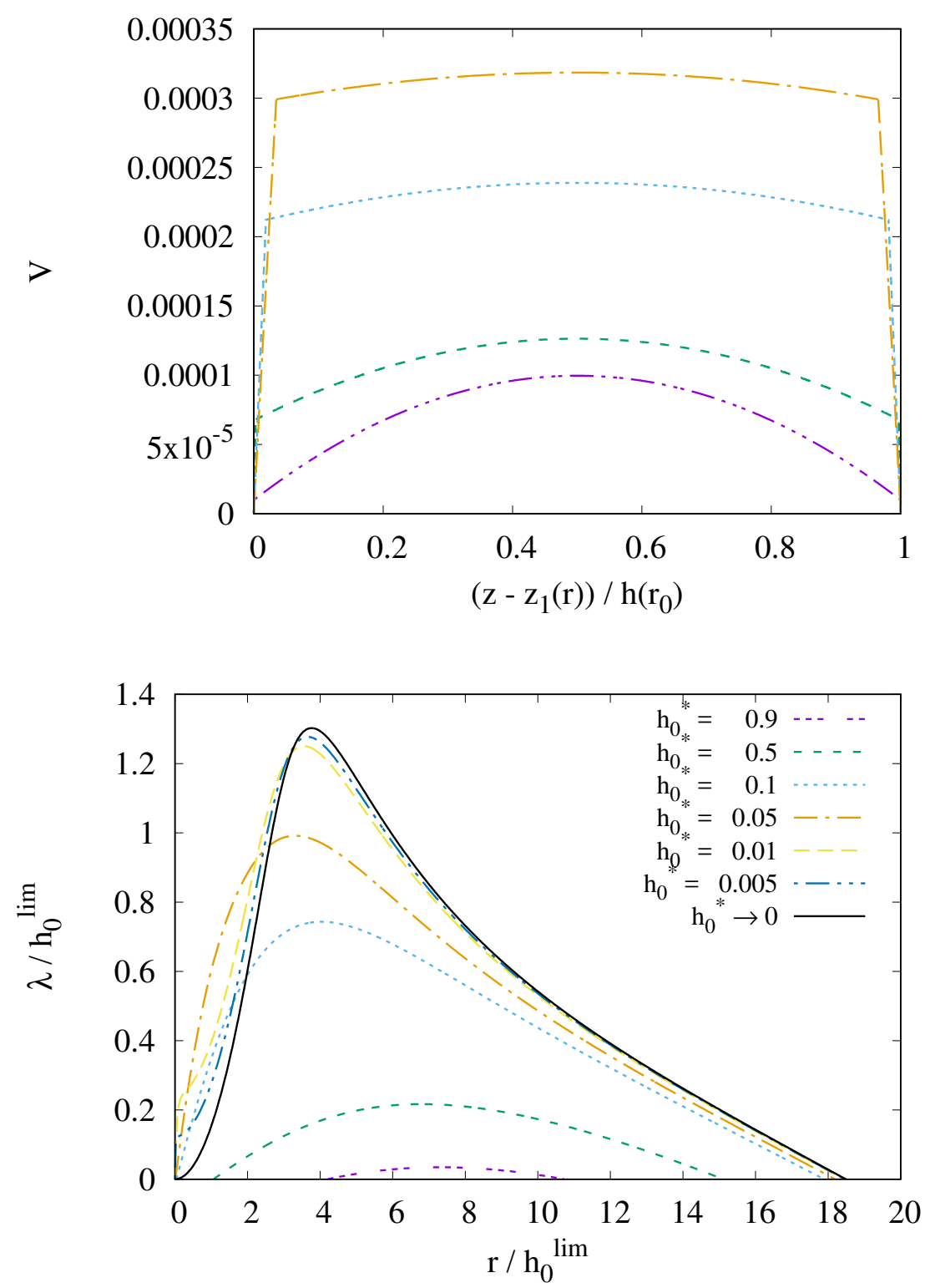

FIG. 3. Top: Velocity profiles for different values of $h_{0}^{*}=h_{0} / h_{0}^{\text {lim }}$ at $r=2 \times 10^{-6} \mathrm{~m} \approx 0.15 h_{0}^{\lim }$. The key is the same than the bottom one. Bottom: The corresponding slip lengths calculated via Eq. (20). The limit of such an equation when $h_{0} \rightarrow 0$ has been also drawn.

position $r=2 \mu \mathrm{m} \approx 0.15 h_{0}^{\text {lim }}$ which is included in the region $\mathcal{R}_{b}$ for all the gaps considered. In Fig. (3) (top) the velocity profiles of the system at different gaps are shown, while in Fig. (3) (bottom) we show the corresponding slip lengths. The slip length in the limit $h_{0} \rightarrow 0$ is 
also shown.

\section{COMPARISON WITH EXPERIMENTS}

\section{A. Hydrodynamic force acting on a sphere approaching a flat surface}

Direct observations of slip from velocity profiles of simple fluids are scarce, with most of them, to the best of our knowledge, being estimated in microchannels or between planar walls [24]. In this section, the analytical expression of the force between spheres in Eqs. (14) and (15) will be compared against the experimental results in Ref. [28] where slip has been inferred indirectly from drag on a sphere. The experiment consists of a sphere of radius $a_{1}=10.4 \mu \mathrm{m}$ immersed in an aqueous sucrose solution and approaching a plane surface $\left(a_{2} \rightarrow \infty\right)$. The control parameter is the approaching velocity $V$ whereas the force on the sphere is measured as a function of the separation distance. For an approaching velocity $V=2400 \mathrm{~nm} / \mathrm{s}$ the experiments show a good agreement with the analytical theory for a Newtonian (Stokes) fluid with no-slip boundary conditions enforced (Fig. 4: blue circles for experiments and blue line for theory). On the other hand, for $V=21600 \mathrm{~nm} / \mathrm{s}$ deviations from the analytical result with no-slip conditions are observed (Fig. 4: red circles).

Such a deviation can be explained by relaxing the Newtonian approximation and letting the fluid to show shear thinning at very high shear rates only. The biviscous model offers a possible route for the explanation of experimental data. From Eq. (7) the critical shear rate can be calculated as

$$
\dot{\gamma}_{c}=V \sqrt{\frac{9 a}{2}\left(\frac{3}{4 h_{0}^{l i m}}\right)^{3}}
$$

From the experimental data with $V=2400 \mathrm{~nm} / \mathrm{s}$, given that the fluid behaves as Newtonian at least until $1 / h=3 \times 10^{7} \mathrm{~m}^{-1}$ one can deduce that $h_{0}^{\lim }<3 \times 10^{-8} \mathrm{~m}$ leading to $\dot{\gamma}_{c}>$ $1800 \mathrm{~s}^{-1}$. On the other hand, from the experimental curve at $V=21600 \mathrm{~nm} / \mathrm{s}$, we consider that deviations from the Newtonian behavior occur around $1 / h \sim 10^{7} \mathrm{~m}^{-1}$ which would give a critical shear rate of $\dot{\gamma}_{c} \sim 3000 \mathrm{~s}^{-1}$. Note that this value for the critical shear rate is also close to that given in Ref. [22] for the onset of slippage effects in a Newtonian fluid. By assuming, for example $\dot{\gamma}_{c}=3500 \mathrm{~s}^{-1}$, the present biviscous model can adjust quite well experimental data by choosing $\eta_{1} / \eta_{0}=0.6$ (Fig. 4: red line). In conclusion, the present biviscous model exhibits a high shear rate shear-thinning behaviour which can be alternatively interpreted as an apparent shear-rate dependent surface slip occurring between two approaching spheres. 


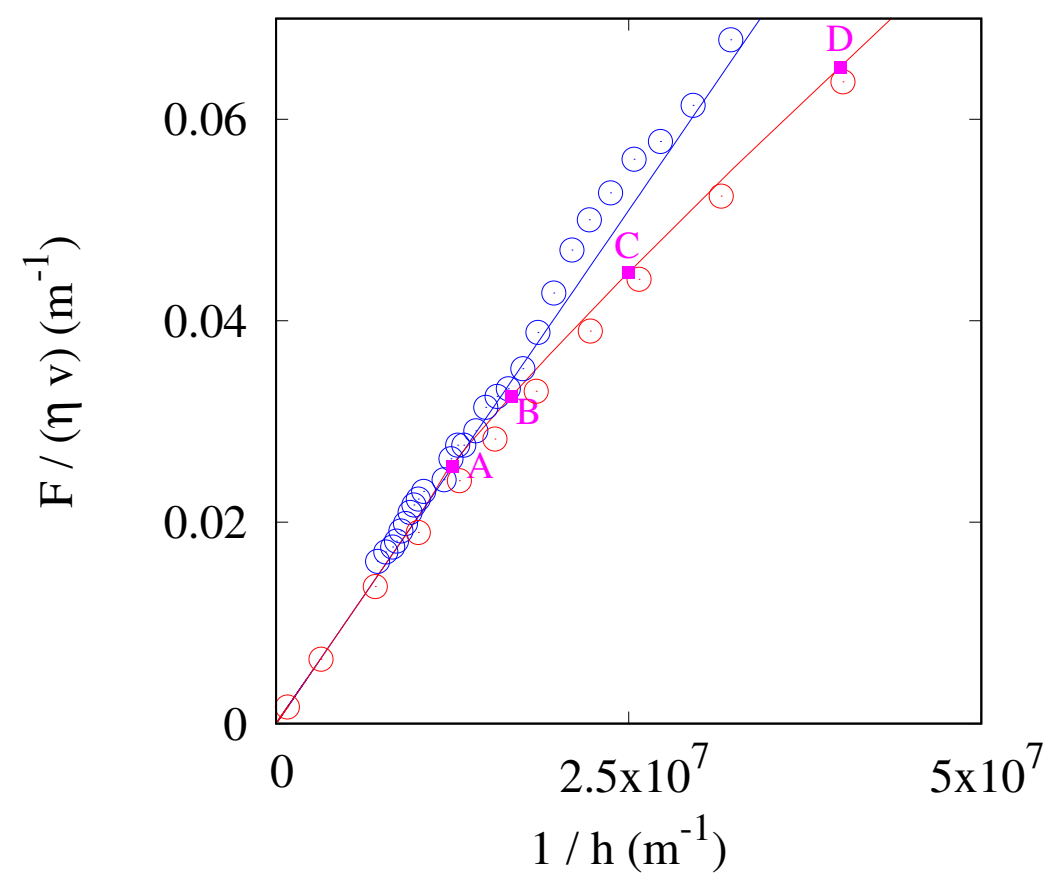

FIG. 4. Force on the approaching sphere as a function of the inverse of the separation between the sphere and the plane surface. The experimental results by Neto et al. [28] have been drawn with circles. For the case of an approaching velocity $2400 \mathrm{~nm} / \mathrm{s}$ the fluid displays Newtonian behavior (blue circles) for the whole range of distances. For the case of an approaching velocity of $21600 \mathrm{~nm} / \mathrm{s}$ (red circles), the Newtonian behavior is observed only at large separations. As the sphere approaches the planar surface, deviations from the Newtonian behaviour are observed. The continuum red line is obtained from the the theoretical result (15) with the following fitting parameters $\dot{\gamma}_{c}=3500 \mathrm{~s}^{-1}$ and $\eta_{1} / \eta_{0}=0.6$. The pink squares A-D correspond to the frames shown at Fig. 5 .

The results are consistent with the experimental data provided by Neto et al. [28]. Shearrate dependence of the slip length was also reported for other Newtonian liquids and was shown to correlate with surface wettability and roughness [20, 21, 40]. It should be noticed that in the case of smoothed hydrophobic surfaces small apparent slip lengths were observed with no shear-rate dependence up to $5000 \mathrm{~s}^{-1}[41]$.

In Fig. 5 the local topology of the interface separating fluid regions with viscosity $\eta_{1}$ (low-viscosity regions: light gray) from those where the viscosity is $\eta_{0}$ (high-viscosity regions: dark gray) is shown. Low-viscosity regions can be interpreted as those where apparent slip 

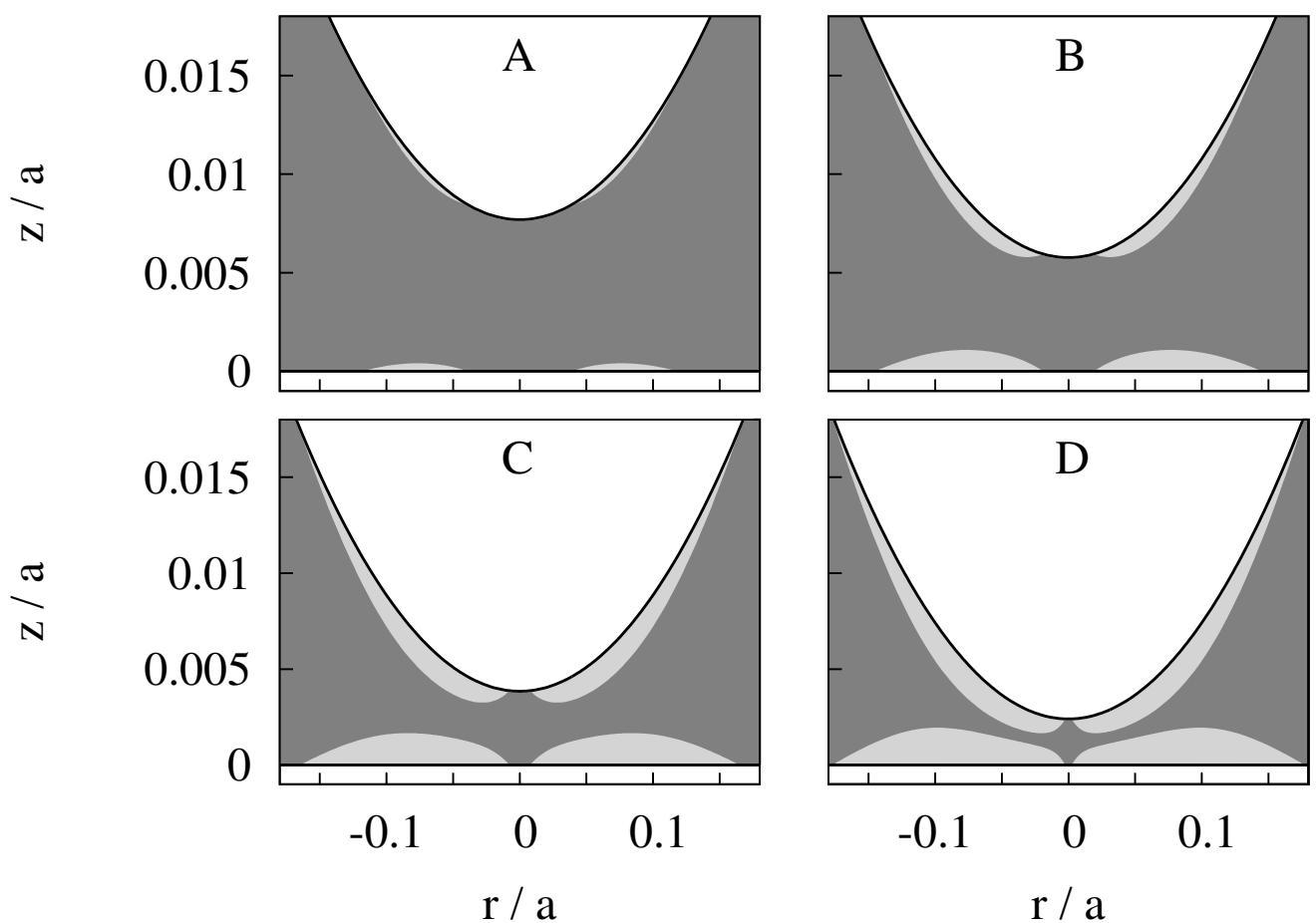

FIG. 5. Low-viscosity regions for the squeezing flow between a spheres of radius $a_{1}=10.4 \mu \mathrm{m}$ and a flat surface for different gaps $h_{0}$. Snapshots A-D correspond to the points in the force highlighted in Fig. 4.

occurs. When the sphere gets closer to the surface, the low-viscosity regions become wider, spreading into the bulk in the limit $h_{0} \rightarrow 0$. Snapshots A-D correspond to the points in the force highlighted in Fig. 4. Bigger deviations arise in correspondence of increased near-wall low-viscosity layers. Deviations from the no-slip Stokes result for approaching particles are expected to become significant for gaps $h_{0}<h_{0}^{\text {lim }}$ at the given macroscopic shear rate.

\section{B. Hydrodynamic force between two spheres: biviscous vs slip models}

In Ref. [22], a phenomenological model has been proposed for the slip length. Even though the model is phenomenological, we compare our results with the result of this model because the latter accounts for experimental results. In this way, we are comparing indirectly with experimental results [3, 4]. The slip length model of Ref. [22] is given by the following 
phenomenological expression

$$
\lambda(\dot{\gamma}(r))=\lambda_{\max }-\frac{\lambda_{\max }-\lambda_{\min }}{1+\frac{\dot{\gamma}(r)}{\dot{\gamma}_{c}}}
$$

and they have performed an inverse-engineering study, i.e. they have adjusted the model parameters to match the STNBS observed in experiments [3, 4]. In the case of Ref. [3], they found that $\lambda_{\min } / a=0, \lambda_{\max } / a=0.04, \dot{\gamma}_{c}=1000 \mathrm{~s}^{-1}$ was reproducing accurately experimental data for the suspension viscosity. In this section we compute the corresponding lubrication force acting between two spheres assuming that a slip governed by Eq.(23) occurred on the particle surface. In order to do it, we start from the expression for the pressure gradient $\partial p(r) / \partial r$ presented in Ref. [22] (see their Eq.(9)-(12)) and double integrate it numerically along the radial coordinate $r$ to derive the force, i.e.

$$
p(r)=-\int_{r}^{\infty} \frac{\partial p}{\partial r}(r) d r
$$

and

$$
F=\int_{0}^{\infty} p(r) 2 \pi r d r
$$

In the solution, we consider an average bulk shear rate $\dot{\gamma}=10 \mathrm{~s}^{-1}$, particle radii $a=$ $43 \mu \mathrm{m}$. Fig. 6 shows the resulting interparticle lubrication force coming from the Kroupa analysis with experimentally-derived model parameters and the lubrication force obtained analytically for the biviscous model, i.e. Eq.(15). In the biviscous model viscosity $\eta_{0}$ is taken to be that of the glycerol solution of Ref. [3]. In addition the critical shear rate $\dot{\gamma}_{c}=1000 \mathrm{~s}^{-1}$ is chosen to be the same as as in the slip model of Ref. [22]. Effect of the only left adjusting parameter in the biviscous model, i.e. the viscosity ratio $\eta_{1} / \eta_{0}$ is also shown in the figure. It is clear that the biviscous model captures well the transition between the far-field behaviour and and the short-range force plateau at the given distance $(\approx 0.1 a)$. For $\eta_{1} / \eta_{0}=0.001$ the agreement with the Kroupa model (matching the experiment) is excellent.

The result shows that, in terms of interparticle lubrication forces, the behaviour of a solvent matrix characterized by "hidden shear-thinning" behaviour at large shear rate [13] is consistent with that one of a perfectly Newtonian matrix with finite slippage at the particle surface. As a consequence, the origin of the STNBS can be interpreted in both ways. 


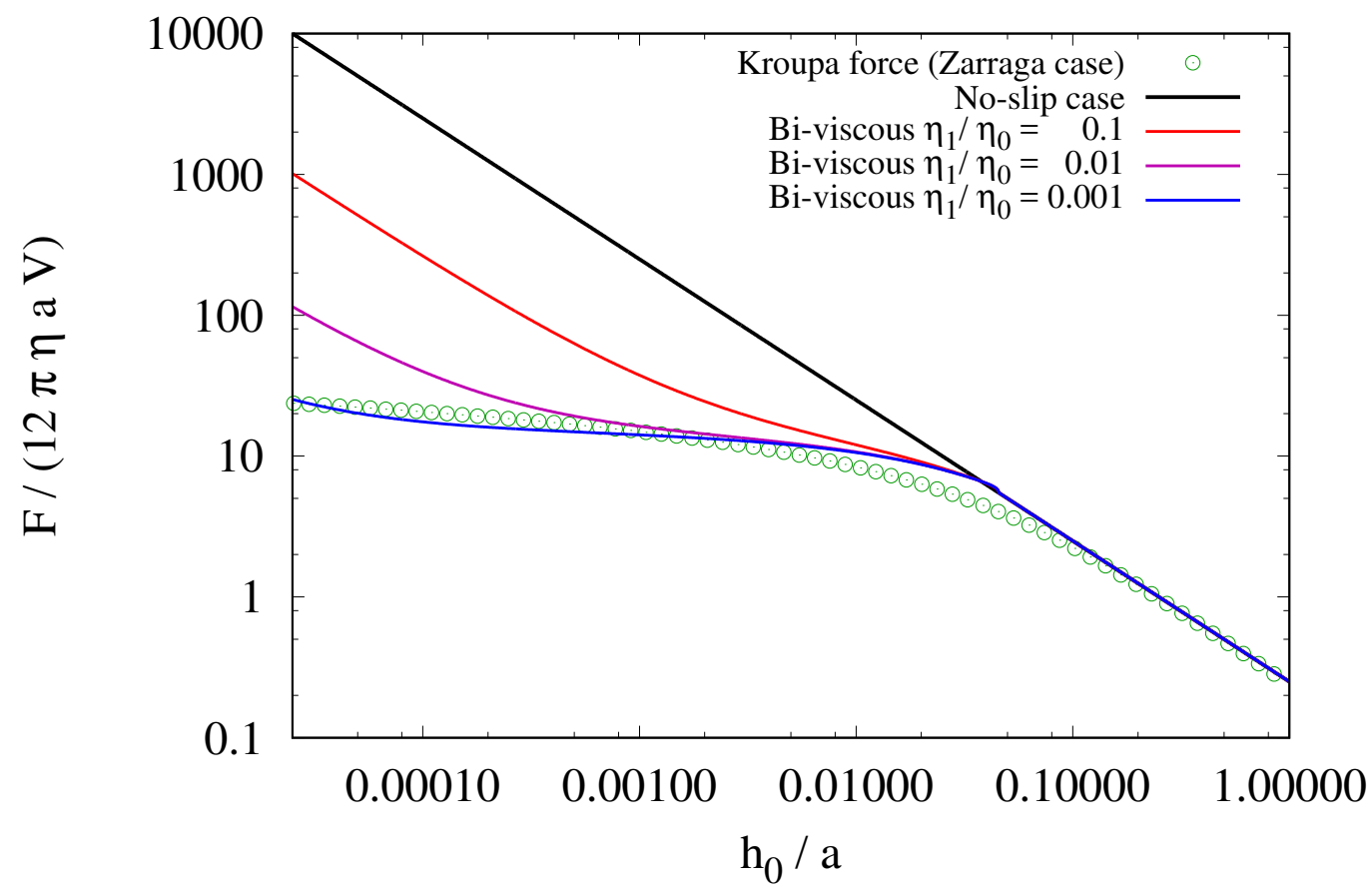

FIG. 6. Comparison of interparticle lubrication forces between two spheres of radius $a=43 \mu \mathrm{m}$ in a shear flow with average $\dot{\gamma}=10 \mathrm{~s}^{-1}$. Black line: Newtonian lubrication. Blue circles: force derived by Kroupa's slip model [22] with experimentally-adjusted parameters $\left(\lambda_{\min } / a=0, \lambda_{\max } / a=0.04\right.$, $\dot{\gamma}_{c}=1000 \mathrm{~s}^{-1}$ ). Red/purple/green lines: biviscous model; $\dot{\gamma}_{c}=1000 \mathrm{~s}^{-1}$.

As a last remark, it should be noticed that the critical shear-rate $\dot{\gamma}_{c}$ in the biviscous model needed to obtain quantitative agreement with the Kroupa's results (and therefore with the experimental data of Ref. [3]) is orders of magnitudes smaller than realistic values for which shear-thinning of the solvent practically occurs, i.e. for glycerol matrices $\dot{\gamma}_{c}$ is expected to be in the order $10^{4}-10^{5} \mathrm{~s}^{-1}$ or even larger [36]. The present low value of $\dot{\gamma}_{c}$ is consistent with experimentally-adjusted wall-slip models and shows that an effective shear-thinning rheology (rather than a true bulk rheology) of the solvent in the narrow gaps between approaching particles can ultimately lead to the observed overall STNBS.

\section{CONCLUSIONS}

In this article the biviscous model has been used to determine the apparent slip between spheres caused by a 'hidden' shear thinning of a nominally-Newtonian fluid, i.e. occurring 
only at high shear rates or as a result of the interaction of the lubricating liquid with the external geometries. We propose to use a critical shear rate $\dot{\gamma}_{c}$ (for the onset of shear-thinning effects) and a high-to-low shear viscosity ratio $\eta_{1} / \eta_{0}$ as the relevant parameters to determine the 'apparent' slip. Differently to current slip length models, where slippage velocity is considered as a boundary condition and used to calibrate experimental results, here the slip length can be uniquely inferred from the rheological parameters of the solvent ( $\dot{\gamma}_{c}$ and $\left.\eta_{1} / \eta_{0}\right)$ which could be estimated through independent rheological or AFM experiments. The biviscous model may describe two physical situations, either a true rheological property of the solvent (as in silicon oils [36]) or an effective viscosity decrease in simpler fluids, e.g. due to wall roughness or near-wall liquid inhomogeneities such as microstructural changes or presence of a dissolved phase [22, 23, 28]. Moreover, we demonstrate that in this biviscous model, the slip length is not constant along the surface but contains a natural shear-rate dependence in agreement with experimental findings [20, 21, 28] and previous suggested theories $[32,33]$. Experimental data on the hydrodynamic drag experienced by a sphere approaching a planar wall are excellently reproduced by the model. In terms of implications on suspension rheology, these results bridge therefore the 'hidden' solvent shear-thinning theory [13] with the slip-based models presented recently in Ref. [22] as a possible explanation of the shear-thinning in hard-sphere non-Brownian suspensions.

The present biviscous model allows also for a natural incorporation of non-continuum nano-rheology effects for lubricating fluids under very confined situations and can pave the way towards more realistic short-range hydrodynamic interaction models between particles in real suspensions.

\section{ACKNOWLEDGMENTS}

The authors wish to thank the Reviewers for the interesting suggestions as well as Roger Tanner for useful discussions. M.E. and A.V-Q acknowledge the financial support provided by the Welsh Government and Higher Education Funding Council for Wales through the Ser Cymru National Research Network in Advanced Engineering and Materials is gratefully acknowledged. Computing resources offered by HPC Wales via the Project No. HPCWT050 (Multiscale particle simulation for complex fluids). A.V-Q and P.E also thanks support from MINECO (Spain) under grants No. FIS2013-47350-C5-1-R, FIS2013-47350-C5-3-R, 
FIS2017-86007-C3-1-P and FIS2017-86007-C3-3-P.

[1] F. Ferrini, D. Ercolani, B. de Cindio, L. Nicodemo, L. Nicolais, and S. Ranaudo, Rheologica Acta 18, 289 (1979).

[2] F. Gadala-Maria and A. Acrivos, Journal of Rheology 24, 799 (1980).

[3] I. E. Zarraga, D. A. Hill, and D. T. Leighton Jr, Journal of Rheology 44, 185 (2000).

[4] S.-C. Dai, E. Bertevas, F. Qi, and R. I. Tanner, Journal of Rheology 57, 493 (2013).

[5] J. Mewis and N. J. Wagner, Colloidal Suspension Rheology (Cambridge University Press, 2011) cambridge Books Online.

[6] G. Chatte, J. Comtet, A. Nigues, L. Bocquet, A. Siria, G. Ducouret, F. Lequeux, N. Lenoir, G. Ovarlez, and A. Colin, Soft Matter 14, 879 (2018).

[7] R. Mari, R. Seto, J. F. Morris, and M. M. Denn, Journal of Rheology 58, 1693 (2014).

[8] J. Bergenholtz, J. F. Brady, and M. Vicic, Journal of Fluid Mechanics 456, 239275 (2002).

[9] A. Sierou and J. F. Brady, Journal of Rheology 46, 1031 (2002).

[10] E. Bertevas, X. Fan, and R. I. Tanner, Rheologica acta 49, 53 (2010).

[11] A. Vázquez-Quesada and M. Ellero, Journal of Non-Newtonian Fluid Mechanics (2016).

[12] S. Dai, , and R. I. Tanner, Rheol Acta 55, 739 (2016).

[13] A. Vázquez-Quesada, R. I. Tanner, and M. Ellero, Phys. Rev. Lett. 117, 108001 (2016).

[14] A. Jabbarzadeh, J. Atkinson, and R. Tanner, Journal of Non-Newtonian Fluid Mechanics 69, 169 (1997).

[15] J. Klein and E. Kumacheva, Science 269, $816 \quad$ (1995), http://science.sciencemag.org/content/269/5225/816.full.pdf.

[16] J. Klein and E. Kumacheva, The Journal of Chemical Physics 108, 6996 (1998).

[17] E. Kumacheva and J. Klein, The Journal of Chemical Physics 108, 7010 (1998).

[18] A. L. Demirel and S. Granick, Phys. Rev. Lett. 77, 2261 (1996).

[19] L. Bureau, Phys. Rev. Lett. 104, 218302 (2010).

[20] Y. Zhu and S. Granick, Phys. Rev. Lett. 87, 096105 (2001).

[21] Y. Zhu and S. Granick, Phys. Rev. Lett. 88, 106102 (2002).

[22] M. Kroupa, M. Soos, and J. Kosek, Phys. Chem. Chem. Phys. 19, 5979 (2017).

[23] O. I. Vinogradova, Langmuir 11, 2213 (1995). 
[24] E. Lauga, M. Brenner, and H. Stone, in Springer handbook of experimental fluid mechanics (Springer, 2007) pp. 1219-1240.

[25] V. Bertola, F. Bertrand, H. Tabuteau, D. Bonn, and P. Coussot, Journal of Rheology 47, 1211 (2003).

[26] S. G. Hatzikiriakos, Progress in Polymer Science 37, 624 (2012), topical Issue on Polymer Physics.

[27] M. M. Denn, Annual Review of Fluid Mechanics 33, 265 (2001).

[28] C. Neto, V. Craig, and D. Williams, The European Physical Journal E 12, 71 (2003).

[29] J.-L. Barrat and L. Bocquet, Phys. Rev. Lett. 82, 4671 (1999).

[30] J. Baudry, E. Charlaix, A. Tonck, and D. Mazuyer, Langmuir 17, 5232 (2001).

[31] D. Savio, L. Pastewka, and P. Gumbsch, Science Advances 2 (2016), 10.1126/sciadv.1501585, http://advances.sciencemag.org/content/2/3/e1501585.full.pdf.

[32] P. G. de Gennes, Langmuir 18, 3413 (2002).

[33] E. Lauga and M. P. Brenner, Phys. Rev. E 70, 026311 (2004).

[34] P. Dontula, C. W. Macosko, and L. E. Scriven, Industrial \& Engineering Chemistry Research 38, 1729 (1999).

[35] C. Pipe, T. Majmudar, and G. McKinley, Rheol Acta 47, 621 (2008).

[36] A. Vázquez-Quesada, A. Mahmud, S. Dai, M. Ellero, and R. I. Tanner, Journal of NonNewtonian Fluid Mechanics 248, 1 (2017).

[37] A. Vázquez-Quesada and M. Ellero, Physics of Fluids 28, 073101 (2016).

[38] O. I. Vinogradova, Langmuir 14, 2827 (1998).

[39] O. I. Vinogradova and G. E. Yakubov, Langmuir 19, 1227 (2003).

[40] E. Bonaccurso, H.-J. Butt, and V. S. J. Craig, Phys. Rev. Lett. 90, 144501 (2003).

[41] C. Cottin-Bizonne, B. Cross, A. Steinberger, and E. Charlaix, Phys. Rev. Lett. 94, 056102 (2005). 